\title{
Conserved charges of minimal massive gravity coupled to scalar field
}

\author{
M. R. Setare ${ }^{\mathrm{a}}$, H. Adami ${ }^{\mathrm{b}}$ \\ Department of Science, University of Kurdistan, Sanandaj, Iran
}

Received: 10 October 2017 / Accepted: 7 February 2018 / Published online: 17 February 2018

(C) The Author(s) 2018. This article is an open access publication

\begin{abstract}
Recently, the theory of topologically massive gravity non-minimally coupled to a scalar field has been proposed, which comes from the Lorentz-Chern-Simons theory (JHEP 06, 113, 2015), a torsion-free theory. We extend this theory by adding an extra term which makes the torsion to be non-zero. We show that the BTZ spacetime is a particular solution to this theory in the case where the scalar field is constant. The quasi-local conserved charge is defined by the concept of the generalized off-shell ADT current. Also a general formula is found for the entropy of the stationary black hole solution in context of the considered theory. The obtained formulas are applied to the BTZ black hole solution in order to obtain the energy, the angular momentum and the entropy of this solution. The central extension term, the central charges and the eigenvalues of the Virasoro algebra generators for the BTZ black hole solution are thus obtained. The energy and the angular momentum of the BTZ black hole using the eigenvalues of the Virasoro algebra generators are calculated. Also, using the Cardy formula, the entropy of the BTZ black hole is found. It is found that the results obtained in two different ways exactly match, just as expected.
\end{abstract}

\section{Introduction}

We know that the pure Einstein-Hilbert gravity in three dimensions exhibits no propagating physical degrees of freedom [2-5]. But adding the gravitational Chern-Simons term produces a propagating massive graviton [6]. The resulting theory is called the topologically massive gravity (TMG). Inclusion of a negative cosmological constant yields a cosmological topologically massive gravity (CTMG). In this case the theory exhibits both gravitons and black holes. Unfortunately, in this model a problem is found with the usual sign for the gravitational constant, which means that the massive

\footnotetext{
a e-mail: rezakord@ipm.ir

b e-mail: hamed.adami@yahoo.com
}

excitations of CTMG carry negative energy. In the absence of a cosmological constant the sign of the gravitational constant can be changed but if $\Lambda<0$ this will give a negative mass to the BTZ black hole, making the existence of a stable ground state doubtful in this model [7]. TMG has a bulk-boundary unitarity conflict. In other words either the bulk or the boundary theory is non-unitary so there is a clash between the positivity of the two Brown-Henneaux boundary charges $c$ and the bulk energies [8]. Recently an interesting version of threedimensional massive gravity was introduced by Bergshoeff et al. [9], dubbed Minimal Massive Gravity (MMG), which has the same minimal local structure as TMG. The MMG model has the same gravitational degree of freedom as the TMG has and the linearization of the metric field equations for the MMG yields a single propagating massive spin-2 field. It seems that the single massive degree of freedom of the MMG is unitary in the bulk and gives rise to an unitary CFT on the boundary. Following this paper some interesting work has been done on the MMG model [10-18].

The authors of [19] have introduced the Chiral Gravity (CG) by formulating the TMG at a special point in parameter space, where the curvature radius of $A d S_{3}$ equals the inverse of the graviton mass $\mu$. Recently the authors of [1] have proposed a generalization of chiral gravity. They have considered a Chern-Simons action for the spin connection in the presence of a scalar field and a constraint that enforces the spin connection to remain torsion-less. So the model includes the TMG and the CG as particular cases. Here we would like to extend the Lagrangian of this model so that it describes minimal massive gravity theory, non-minimally coupled to a scalar field. There are many papers about the coupling of the scalar field and gravity in three dimensions. For example, the contribution of the scalar fields to the conserved charges has been studied in the context of black hole solutions of 3D gravity with a scalar field in [20]. The authors in this paper have shown that although the generators of the asymptotic symmetries acquire a contribution from the scalar 
field, the asymptotic symmetry group remains the same as in pure gravity. In Ref. [21] the author has considered the three-dimensional gravity coupled to a scalar field, with special attention to black hole configurations. A finite action for three-dimensional gravity with a minimally coupled selfinteracting scalar field has been constructed in [22]. In [23] a spinning hairy black hole in gravity, minimally coupled to a self-interacting real scalar field in three spacetime dimensions, has been presented. The authors in this paper have shown that the presence of a scalar field with a slower fall-off at infinity leads to anti-de Sitter asymptotic behavior, which differs from the one found by Brown and Henneaux but has the same symmetry group as in pure gravity.

In this paper we try to find a general formula for a quasilocal conserved charge for our model in a first order formalism. We use the formula obtained to find the energy, the angular momentum and the entropy of the BTZ black hole solution in the context of this theory. It is interesting that although the conserved charges (energy, angular momentum and entropy) of a BTZ black hole and also a Virasoro central charge depend on the coupling parameter $\alpha$ in $\mathrm{MMG}$ [24], the contributions from the MMG interaction term in the energy, angular momentum entropy and also Virasoro central charge vanish for the solution we consider (the BTZ black hole solution in the presence of constant scalar field). It seems that for this special solution case, the coupling to the scalar field removes the dependence on $\alpha$.

There are several approaches to obtain mass and angular momentum of black holes for different gravity theories [2-41]. The authors of [28] have obtained the quasilocal conserved charges for black holes in any diffeomorphically invariant theory of gravity. By considering an appropriate variation of the metric they have established a oneto-one correspondence between the ADT approach and the linear Noether expressions. They have extended this work to a theory of gravity containing a gravitational ChernSimons term in [29] and have computed the off-shell potential and quasi-local conserved charges of some black holes in TMG.

In the metric formalism of gravity for the covariant theories defined by a Lagrangian $n$-form $L$ Wald has shown that the entropy of black holes is the Noether charge associated with the horizon-generating Killing vector field, evaluated at the bifurcation surface [25]. The presence of the purely gravitational Chern-Simons terms and mixed gauge gravitational ones gives rise to a non-covariant theory of gravity in the metric formalism. Tachikawa extended the Wald approach to include non-covariant theories [42]. Hence, regarding this extension, one can obtain the black hole entropy as a Noether charge in the context of non-covariant theories as well. Another way (apart from the Tachikawa method) to obtain the entropy of black holes in the context of such theories has been studied in Refs. $[43,44]$ in an appropriate way.
The remainder of this paper is organized as follows. In Sect. 2 we first briefly introduce the model of [1]. Then, by adding a convenient term to the Lagrangian of [1], this model is generalized, such that it describes a minimal massive gravity theory, non-minimally coupled to a scalar field. In contrast to the Lagrangian of [1] this model is not torsion-free. In Sect. 3 the equations of motion are obtained. It is shown that the new field $h$ is not a symmetric tensor, in contrast to the usual minimal massive gravity. Also it is shown that the BTZ black hole spacetime solves the equations of motion. In Sect. 4 an expression for the conserved charges of the considered model is found, associated with the asymptotic Killing vector field $\xi$ based on the quasi-local formalism. In Sect. 5 a stationary black hole solution of the minimal massive gravity coupled to a scalar field is considered. Then a general formula for entropy of such black hole solutions is found. In Sect. 6 the obtained formula is applied to conserved charges and the entropy on the BTZ black hole solution of the minimal massive gravity coupled to a scalar field model. The energy, angular momentum and entropy of these black holes are obtained. In Sect. 7, the central extension term is calculated and with this we find the central charges and the eigenvalues of the Virasoro algebra generators for the BTZ black hole solution. Also, we obtain again the energy and angular momentum of this black hole using the eigenvalues of the Virasoro algebra generators. Then the entropy of the BTZ black hole is found using the Cardy formula. In the final section the results are summarized.

\section{Minimal massive gravity coupled to a scalar field}

Recently it has been shown that it is possible to achieve from the Lorentz-Chern-Simons action a topologically massive gravity (TMG), non-minimally coupled to a scalar field [1]. The Lorentz-Chern-Simons Lagrangian 3-form is

$L_{C S}(\omega)=\omega^{a}{ }_{b} \wedge \mathrm{d} \omega^{b}{ }_{a}+\frac{2}{3} \omega^{a}{ }_{b} \wedge \omega^{b}{ }_{c} \wedge \omega^{c}{ }_{a}$,

where $\omega^{a b}=\omega^{a b}{ }_{\mu} \mathrm{d} x^{\mu}$ are the components of the spinconnection 1 -form. ${ }^{1}$ The spin connection can be decomposed in two independent parts,

$\omega^{a b}=\tilde{\omega}^{a b}+K^{a b}$,

where $\tilde{\omega}^{a b}$ is the torsion-free part, which is known as the Riemannian spin connection and $K^{a b}$ is the contorsion 1-

\footnotetext{
${ }^{1}$ Here, we use Latin and Greek Letters to characterize Lorentz and coordinate indices, respectively. Also, we work in the Einstein-Cartan formalism. In this formalism the independent dynamical fields are the spin connection $\omega^{a b}$ and the dreibein 1-form $e^{a}$.
} 
form. The field equations for the Lorentz-Chern-Simons Lagrangian are

$R^{a b}(\omega)=\mathrm{d} \omega^{a b}+\omega^{a}{ }_{c} \wedge \omega^{c b}=0$,

where $R^{a b}(\omega)$ is the curvature 2-form. Using the Bianchi identities

$D(\omega) R^{a b}(\omega)=0, \quad D(\omega) T^{a}(\omega)=R_{b}^{a}(\omega) \wedge e^{b}$,

we find that $D(\omega) T^{a}(\omega)=0$. This equation has the following solution in three dimensions:

$T^{a}(\omega)=\varphi_{0} \varepsilon^{a}{ }_{b c} e^{b} \wedge e^{c}$,

where $\varphi_{0}$ is a constant. Notice that $D(\omega)$ denotes an exterior covariant derivative with respect to $\omega$ and that $T^{a}(\omega)$ is a torsion 2-form which is defined as

$T^{a}(\omega)=D(\omega) e^{a}=\mathrm{d} e^{a}+\omega^{a}{ }_{b} \wedge e^{b}$.

Also, $e^{a}=e^{a}{ }_{\mu} \mathrm{d} x^{\mu}$ are vector valued 1-forms such that $e^{a}{ }_{\mu}$ denotes a dreibein. We shall assume that the dreibein is invertible. Since $T^{a}(\omega)=K_{b}^{a} \wedge e^{b}$ we find that

$K^{a b}=-\varphi_{0} \varepsilon^{a b}{ }_{c} e^{c}$.

In the paper [1] the authors promote $\varphi_{0}$ to a local dynamical field $\varphi=\varphi(x)$. By substituting Eq. (2) with $K^{a b}=-\varphi \varepsilon^{a b}{ }_{c} e^{c}$ into the Lagrangian (1) we have

$$
\begin{aligned}
L_{C S}(\omega)= & \frac{1}{2} L_{C S}(\tilde{\omega})+\varphi \varepsilon_{a b c} e^{a} \wedge R^{b c}(\tilde{\omega}) \\
& +\frac{1}{3} \varphi^{3} \varepsilon_{a b c} e^{a} \wedge e^{b} \wedge e^{c} \\
& +\varphi^{2} e_{a} \wedge T^{a}(\tilde{\omega})+\frac{1}{2} \mathrm{~d}\left(\varphi \varepsilon_{a b c} \tilde{\omega}^{a b} \wedge e^{c}\right) .
\end{aligned}
$$

Eventually, the authors of Ref. [1] present the following Lagrangian:

$$
\begin{aligned}
L_{[\lambda, m]}= & \varphi \varepsilon_{a b c} e^{a} \wedge R^{b c}(\tilde{\omega})+\frac{1}{3 !} \lambda \varphi^{3} \varepsilon_{a b c} e^{a} \wedge e^{b} \wedge e^{c} \\
& +\frac{1}{2 m} L_{C S}(\tilde{\omega}) \\
& +\varphi^{2} e_{a} \wedge T^{a}(\tilde{\omega})+\frac{1}{2} \mathrm{~d}\left(\varphi \varepsilon_{a b c} \tilde{\omega}^{a b} \wedge e^{c}\right) \\
& +\frac{1}{2 m} \zeta_{a} \wedge T^{a}(\tilde{\omega}),
\end{aligned}
$$

where $\lambda$ and $m$ are two parameters that are introduced to adapt the cosmological constant and the mass parameter of the TMG term, respectively. The last term in the Lagrangian (9) makes this theory torsion free. The above
Lagrangian describes a topologically massive gravity theory non-minimally coupled to a scalar field.

In three dimensions it is convenient to define a dualized spinconnection 1-form and a dualized curvature 2-form

$\omega^{a}=\frac{1}{2} \varepsilon^{a}{ }_{b c} \omega^{b c}, \quad R^{a}=\frac{1}{2} \varepsilon^{a}{ }_{b c} R^{b c}$,

respectively. By using a 3D-vector algebra notation for Lorentz vectors (see for instance [45]) the dualized curvature and torsion 2 -forms can be written as

$R=\mathrm{d} \omega+\frac{1}{2} \omega \times \omega, \quad T=\mathrm{d} e+\omega \times e$,

in terms of a dualized spin-connection, respectively.

So far we have reviewed the main idea of the paper [1]. Now the Lagrangian (9) will be generalized in order to describe the minimal massive gravity theory, non-minimally coupled to a scalar field. ${ }^{2}$ First of all, we consider the following redefinitions in the Lagrangian (9):

$\lambda \rightarrow 2 \lambda, \quad m \rightarrow \mu, \quad L_{[\lambda, m]} \rightarrow 2 L$,

$\frac{1}{4 m} \zeta \rightarrow h, \quad \tilde{\omega} \rightarrow \omega$,

and then we add the following term to the obtained Lagrangian:

$\frac{1}{2} \alpha e \cdot h \times h$,

where $\alpha$ is just a parameter with a dimension of mass inverted. Thus we obtain the following Lagrangian:

$$
\begin{aligned}
L= & \varphi e \cdot R(\omega)+\frac{1}{3 !} \lambda \varphi^{3} e \cdot e \times e \\
& +\frac{1}{2 \mu}\left(\omega \cdot \mathrm{d} \omega+\frac{1}{3} \omega \cdot \omega \times \omega\right) \\
& +\frac{1}{2} \varphi^{2} e \cdot T(\omega)+h \cdot T(\omega) \\
& +\frac{1}{2} \mathrm{~d}(\varphi \omega \cdot e)+\frac{1}{2} \alpha e \cdot h \times h .
\end{aligned}
$$

It is easy to see that the theory described by the above Lagrangian is not a torsion-free one (for 3D gravity models with torsion refer to [47-49]). If the two last terms in the Lagrangian (14) are eliminated, the obtained Lagrangian can be interpreted as a Lagrangian of the Mielke-Baekler model [46], non-minimally coupled to a scalar field. However, we

\footnotetext{
${ }^{2}$ In order to generalize the Lagrangian (9), we consider the spin connection $\tilde{\omega}^{a b}$ and the dreibein $e^{a}$ as two independent dynamical fields on an equal footing.
} 
should note that the Mielke-Baekler Lagrangian is given by [46]

$$
\begin{aligned}
L_{M B}= & \theta_{C} e \cdot R(\omega)+\theta_{\Lambda} e \cdot e \times e+\theta_{L} \\
& \times\left(\omega \cdot \mathrm{d} \omega+\frac{1}{3} \omega \cdot \omega \times \omega\right)+\theta_{T} e \cdot T(\omega)+h \cdot T(\omega),
\end{aligned}
$$

where $\theta_{C}, \theta_{\Lambda}, \theta_{L}$ and $\theta_{T}$ are just constants. By comparing Eqs. (14) and (15), one may guess that, by changing the frame as $e \rightarrow \varphi e$, the Mielke-Baekler Lagrangian (15) turns into the Lagrangian (14), without the two last terms. But this is not correct because the torsion 2-form will be changed as $T(\omega) \rightarrow T(\omega)+\mathrm{d} \varphi$ e under the considered change of frame. Thus it seems that the considered model, which is described by the Lagrangian (14), cannot be simply seen as a change of frame of the Mielke-Baekler theory.

As will be discussed in Sect. 4, $e$ and $\omega$ both are invariant under a general coordinate transformation. Also, $\varphi$ is a scalar field and therefore the Lagrangian (14) is invariant under a general coordinate transformation. On the other hand, $e$ is invariant under a general Lorenz gauge transformation, $e^{a} \rightarrow \Lambda_{b}^{a} e^{b}$, but $\omega$ is not, $\omega \rightarrow \Lambda \omega \Lambda^{-1}+\Lambda \mathrm{d} \Lambda^{-1}$. It is easy to check that the curvature 2 -form and the torsion 2 -form both are invariant under the general Lorenz gauge transformation. So the Lagrangian (14) is not invariant under the general Lorenz gauge transformation due to the presence of the topological Chern-Simons term (the third term in the Lagrangian). Thus, in order to obtain the conserved charges of such a theory, we will have to use an extension of the Tachikawa method [42], which is presented in $[31,50]$. We assume that $h^{a}{ }_{\mu} \mathrm{d} x^{\mu}$ is a Lorentz vector-valued 1-form.

\section{Equations of motion}

To find the equations of motion, a variation of the Lagrangian (14) should be taken,

$\delta L=\delta \varphi E_{\varphi}+\delta e \cdot E_{e}+\delta \omega \cdot E_{\omega}+\delta h \cdot E_{h}+\mathrm{d} \Theta(\Phi, \delta \Phi)$

where $\Phi$ is a collection of all the fields, i.e. $\Phi=\{\varphi, e, \omega, h\}$. In the above equation the following definitions are valid:

$$
\begin{aligned}
E_{\varphi}= & e \cdot R(\omega)+\frac{\lambda}{2} \varphi^{2} e \cdot e \times e+\varphi e \cdot T(\omega), \\
E_{e}= & \varphi R(\omega)+\frac{\lambda}{2} \varphi^{3} e \times e+\frac{1}{2} \varphi^{2} T(\omega)+\frac{1}{2} \alpha h \times h \\
& +\frac{1}{2} D(\omega)\left(\varphi^{2} e\right)+D(\omega) h, \\
E_{\omega}= & \frac{1}{\mu} R(\omega)+\frac{1}{2} \varphi^{2} e \times e+e \times h+D(\omega)(\varphi e),
\end{aligned}
$$

$$
E_{h}=T(\omega)+\alpha e \times h,
$$

$$
\begin{gathered}
\Theta(\Phi, \delta \Phi)=\varphi \delta \omega \cdot e+\frac{1}{2 \mu} \delta \omega \cdot \omega+\frac{1}{2} \varphi^{2} \delta e \cdot e+\delta e \cdot h \\
+\frac{1}{2} \delta(\varphi \omega \cdot e) .
\end{gathered}
$$

The equations of motion of the considered theory are

$E_{\varphi}=E_{e}=E_{\omega}=E_{h}=0$,

and $\Theta(\Phi, \delta \Phi)$ is just a surface term. The equation of motion (20), namely $E_{h}=0$, can be written as

$T(\omega)=\mathrm{d} e+(\omega+\alpha h) \times e=0$.

It is clear that a new dual spin-connection 1-form can be defined:

$\Omega=\omega+\alpha h$,

which is the usual torsion-free spin connection $\Omega=\Omega(e)$. By this definition the equations of motion can be rewritten

$$
\begin{aligned}
& e \cdot R(\Omega)+\frac{\lambda}{2} \varphi^{2} e \cdot e \times e-\alpha \varphi e \cdot e \times h \\
& \quad+\frac{1}{2} \alpha^{2} e \cdot h \times h-\alpha e \cdot D(\Omega) h=0, \\
& \varphi R(\Omega)+\frac{\lambda}{2} \varphi^{3} e \times e-\alpha \varphi^{2} e \times h-\frac{1}{2} \alpha(1-\alpha \varphi) h \times h \\
& \quad+(1-\alpha \varphi) D(\Omega) h+\varphi \mathrm{d} \varphi e=0, \\
& R(\Omega)+\frac{1}{2} \mu \varphi^{2} e \times e+\mu(1-\alpha \varphi) e \times h-\alpha D(\Omega) h \\
& \quad+\frac{1}{2} \alpha^{2} h \times h+\mu \mathrm{d} \varphi e=0, \\
& T(\Omega)=0 .
\end{aligned}
$$

To obtain the above equations the following equation is used:

$D(\omega) f=D(\Omega) f-\alpha h \times f$,

where $f$ is an arbitrary Lorentz vector-valued 1-form. By combining Eqs. (26) and (27) we find

$$
\begin{aligned}
& R(\Omega)+\frac{1}{2}[\alpha \lambda \varphi+\mu(1-\alpha \varphi)] \varphi^{2} e \times e \\
& \quad+\left[\mu(1-\alpha \varphi)^{2}-\alpha^{2} \varphi^{2}\right] e \times h \\
& \quad+[\alpha \varphi+\mu(1-\alpha \varphi)] d \varphi e=0 .
\end{aligned}
$$


This equation can be solved to find the following expression for $h$

$$
\begin{aligned}
h_{\mu}^{a}=- & \frac{1}{\left[\mu(1-\alpha \varphi)^{2}-\alpha^{2} \varphi^{2}\right]}\left\{S^{a}{ }_{\mu}\right. \\
& +\frac{1}{2}[\alpha \lambda \varphi+\mu(1-\alpha \varphi)] \varphi^{2} e^{a}{ }_{\mu} \\
& \left.+[\alpha \varphi+\mu(1-\alpha \varphi)] \varepsilon^{a b}{ }_{c} e_{b}{ }^{v} e^{c}{ }_{\mu} \partial_{\nu} \varphi\right\} .
\end{aligned}
$$

In contrast to ordinary minimal massive gravity [9], $h_{\mu \nu}$ is not a symmetric tensor, i.e. in the considered model the condition $e \cdot h=0$ no longer holds. In Eq. (31), $S_{\mu \nu}=\mathcal{R}_{\mu \nu}-\frac{1}{4} g_{\mu \nu} \mathcal{R}$ is the $3 \mathrm{D}$ Schouten tensor and $\mathcal{R}_{\mu \nu}$ and $\mathcal{R}$ are the Ricci tensor and the Ricci scalar, respectively.

It can be easily seen from Eq. (31) that $h^{a}$ is a Lorentz vector-valued 1-form. As we mentioned earlier, dreibein, curvature 2- form and torsion 2-form are invariant under general coordinate transformations (and under general Lorenz gauge transformation). Also, as $D(\Omega)$ is an exterior covariant derivative with respect to $\Omega$, the equations of motion (25)-(28) are covariant.

For BTZ black hole spacetime [51], we find

$$
R(\Omega)=-\frac{1}{2 l^{2}} e \times e, \quad S^{a}=-\frac{1}{2 l^{2}} e^{a},
$$

where $l$ is the AdS space radius. By assuming that $\varphi$ is a constant, say $\varphi=\varphi_{0}$, the BTZ black hole spacetime solves the equations of motion (25)-(28). So, by taking $\varphi=\varphi_{0}$ for the BTZ black hole spacetime, Eq. (31) becomes

$h^{a}=\beta e^{a}$,

where

$\beta=\frac{1-\alpha \lambda l^{2} \varphi_{0}^{3}-\mu l^{2}\left(1-\alpha \varphi_{0}\right) \varphi_{0}^{2}}{2 l^{2}\left[\mu\left(1-\alpha \varphi_{0}\right)^{2}-\alpha^{2} \varphi_{0}^{2}\right]}$.

By substituting Eqs. (32) and (33) into the equations of motion (25)-(28), we find

$$
\begin{aligned}
-\frac{1}{2 l^{2}}+\frac{1}{2} \lambda \varphi_{0}^{2}-\alpha \beta \varphi_{0}+\frac{1}{2} \alpha^{2} \beta^{2} & =0, \\
-\frac{\varphi_{0}}{2 l^{2}}+\frac{1}{2} \lambda \varphi_{0}^{3}-\alpha \beta \varphi_{0}^{2}-\frac{1}{2} \alpha \beta^{2}\left(1-\alpha \varphi_{0}\right) & =0, \\
-\frac{1}{2 l^{2}}+\frac{1}{2} \mu \varphi_{0}^{2}+\mu \beta\left(1-\alpha \varphi_{0}\right)+\frac{1}{2} \alpha^{2} \beta^{2} & =0 .
\end{aligned}
$$

It is obvious that by combining Eqs. (36) and (37) Eq. (34) can be regained. Thus the BTZ black hole spacetime together with $\varphi=\varphi_{0}$ will be a solution of the considered model when Eq. (35) is satisfied, where $\beta$ is given by Eq. (34). When Eqs. (35) and (36) are combined it is found that

$\alpha \beta=0, \quad \varphi_{0}= \pm \frac{1}{l \sqrt{\lambda}}$.
By substituting Eq. (38) into Eq. (37) we obtain

$\beta=\frac{1}{2 l^{2}}\left(\frac{1}{\mu}-\frac{1}{\lambda}\right)$.

Now we have two types of solutions. One solution is

$\alpha=0, \quad \varphi_{0}= \pm \frac{1}{l \sqrt{\lambda}}, \quad \beta=\frac{1}{2 l^{2}}\left(\frac{1}{\mu}-\frac{1}{\lambda}\right)$,

and the other is given by

$\alpha \neq 0, \quad \varphi_{0}= \pm \frac{1}{l \sqrt{\lambda}}, \quad \beta=0, \quad \mu=\lambda$

In both cases we have $T(\omega)=0$, i.e. the BTZ black hole spacetime together with $\varphi=\varphi_{0}$ will be a torsion-free solution of the considered model when one set of Eqs. (40) and (41), are satisfied.

A few comments are necessary. Minimal massive gravity is an extension of topologically massive gravity. Topologically massive gravity is a torsion-free theory but minimal massive gravity is not. A term such as (13) is added to the TMG in order to construct minimal massive gravity. In Sect. 2 it was seen that the Lagrangian (9) describes a topologically massive gravity, non-minimally coupled to a scalar field. In an ordinary case we expect that minimal massive gravity, non-minimally coupled to a scalar field, can be constructed by adding a term like (13) to the Lagrangian (9). The equations of motion of the minimal massive gravity, non-minimally coupled to a scalar, are given by Eqs. (25)-(28). These equations are very complicated because of the complex form of the auxiliary field $h^{a}$ (see Eq. (31)). Therefore writing the equations of motion in metric form is very difficult. Also, it is not useful.

\section{Quasi-local conserved charges}

In this section an expression to the conserved charges of the considered model associated with the asymptotic Killing vector field $\xi$, based on quasi-local formalism for conserved charges, will be found [28-32].

Under the Lorenz gauge transformation $\Lambda \in S O(2,1)$, dreibein transforms according to $e^{a}{ }_{\mu} \rightarrow \Lambda^{a}{ }_{b} e^{b}{ }_{\mu}$ so that the spacetime metric $g_{\mu \nu}=\eta_{a b} e^{a}{ }_{\mu} e^{b}{ }_{\nu}$ under this transformation remains unchanged. Also, under a Lorenz gauge transformation the spin connection transforms according to $\omega \rightarrow \Lambda \omega \Lambda^{-1}+\Lambda \mathrm{d} \Lambda^{-1}$. This is not an invariant quantity under the considered transformation. The Lorentz-Lie (L-L) derivative of the dreibein 1-form can be defined by [52]

$\mathfrak{L}_{\xi} e^{a}=\mathfrak{E}_{\xi} e^{a}+\lambda^{a}{ }_{b} e^{b}$, 
where $\mathfrak{f}_{\xi}$ denotes an ordinary Lie derivative along $\xi$ and $\lambda^{a}{ }_{b}$ generates the Lorenz gauge transformations $S O(2,1)$. In general, $\lambda^{a}{ }_{b}$ is independent of the dynamical fields of the considered model. It is a function of the spacetime coordinates and of the diffeomorphism generator $\xi$. The total variation of the dreibein and the spin connection are defined by [50]

$$
\begin{aligned}
\delta_{\xi} e^{a} & =\mathfrak{L}_{\xi} e^{a}, \\
\delta_{\xi} \omega & =\mathfrak{L}_{\xi} \omega-\mathrm{d} \chi_{\xi},
\end{aligned}
$$

respectively, where $\chi_{\xi}^{a}=\frac{1}{2} \varepsilon^{a}{ }_{b c} \lambda^{b c}$. The extra term in (44), $-\mathrm{d} \chi_{\xi}$, can make a theory non-covariant, in the sense of the Lorentz covariance (because $e$ and $\omega$ both are invariant under a general coordinate transformation).

Now, it is supposed that the variation of the Lagrangian Eq. (16) is due to a diffeomorphism which is generated by the vector field $\xi$, so the total variation of Lagrangian (16) with respect to the diffeomorphism $\xi$ is

$\delta_{\xi} L=\delta_{\xi} \varphi E_{\varphi}+\delta_{\xi} e \cdot E_{e}+\delta_{\xi} \omega \cdot E_{\omega}+\delta_{\xi} h \cdot E_{h}+\mathrm{d} \Theta\left(\Phi, \delta_{\xi} \Phi\right)$.

On the one hand, the presence of the topological ChernSimons term in the Lagrangian (14) makes this model Lorentz non-covariant, by virtue of Eq. (44). So, the total variation of the Lagrangian (14) due to the diffeomorphism generator $\xi$ can be written as

$\delta_{\xi} L=\mathfrak{L}_{\xi} L+\mathrm{d} \psi_{\xi}$,

which is equivalent to the statement that a symmetry is a transformation which leaves the Lagrangian form invariant, up to a total derivative. Despite the fact that a Lagrangian is not invariant under general coordinates transformation and/or under a general Lorenz gauge transformation, if the total variation of a given Lagrangian can be written as (46), then $\xi$ could be a symmetry generator. Although the Lagrangian (14) is not invariant under the general Lorenz gauge transformation, it is invariant under the infinitesimal Lorenz gauge transformation (see Eqs. (46) and (63)) and also a general coordinate transformation. Also, it is enough to obtain generally covariant equations of motion so that the Lagrangian behaves like (46) under a total variation [42]. On the other hand, from the definition of the total variation due to $\xi$, Eqs. (43) and (44)can be written

$$
\begin{aligned}
\delta_{\xi} e & =D(\omega) i_{\xi} e+i_{\xi} T(\omega)+\left(\chi_{\xi}-i_{\xi} \omega\right) \times e, \\
\delta_{\xi} \omega & =i_{\xi} R(\omega)+D(\omega)\left(i_{\xi} \omega-\chi_{\xi}\right), \\
\delta_{\xi} h & =D(\omega) i_{\xi} h+i_{\xi} D(\omega) h+\left(\chi_{\xi}-i_{\xi} \omega\right) \times h, \\
\delta_{\xi} \varphi & =i_{\xi} D(\omega) \varphi,
\end{aligned}
$$

where $i_{\xi}$ denotes the interior product in $\xi$. By substituting Eqs. (46)-(50) into Èq. (45) we find

$$
\begin{aligned}
\mathrm{d}[ & \Theta\left(\Phi, \delta_{\xi} \Phi\right)-i_{\xi} L-\psi_{\xi}+i_{\xi} e \cdot E_{e}+\left(i_{\xi} \omega-\chi_{\xi}\right) \cdot E_{\omega} \\
& \left.+i_{\xi} h \cdot E_{h}\right] \\
= & \left(i_{\xi} \omega-\chi_{\xi}\right) \cdot\left[D(\omega) E_{\omega}+e \times E_{e}+h \times E_{h}\right] \\
& +i_{\xi} e \cdot D(\omega) E_{e}+i_{\xi} h \cdot D(\omega) E_{h} \\
& -i_{\xi} T(\omega) \cdot E_{e}-i_{\xi} R(\omega) \cdot E_{\omega} \\
& -i_{\xi} D(\omega) h \cdot E_{h}-E_{\varphi} i_{\xi} D(\omega) \varphi .
\end{aligned}
$$

The right hand side of the above equation becomes zero by virtue of the Bianchi identities (4). Therefore, we find that

$d J_{\xi}=0$,

where

$J_{\xi}=\Theta\left(\Phi, \delta_{\xi} \Phi\right)-i_{\xi} L-\psi_{\xi}+i_{\xi} e \cdot E_{e}+\left(i_{\xi} \omega-\chi \xi\right) \cdot E_{\omega}+i_{\xi} h \cdot E_{h}$.

Thus the quantity $J_{\xi}$ defined above is conserved off-shell. Because $J_{\xi}$ is closed, it is by virtue of the Poincaré lemma exact so that we can write $J_{\xi}=d K_{\xi}$. Since this model is not Lorentz covariant we expect that the total variation of the surface term differs from its $\mathrm{L}-\mathrm{L}$ derivative

$\delta_{\xi} \Theta(\Phi, \delta \Phi)=\mathfrak{L}_{\xi} \Theta(\Phi, \delta \Phi)+\Pi_{\xi}$.

Now, we take an arbitrary variation from Eq. (53) and we find that

$\mathfrak{J}_{\mathrm{ADT}}(\Phi, \delta \Phi ; \xi)=d\left[\delta K_{\xi}-i_{\xi} \Theta(\Phi, \delta \Phi)\right]+\delta \psi_{\xi}-\Pi_{\xi}$,

where $\mathfrak{J}_{\mathrm{ADT}}(\Phi, \delta \Phi ; \xi)$ is defined as

$$
\begin{aligned}
\mathfrak{J}_{\mathrm{ADT}}(\Phi, \delta \Phi ; \xi)= & \delta e \cdot i_{\xi} E_{e}+\delta \omega \cdot i_{\xi} E_{\omega}+\delta h \cdot i_{\xi} E_{h}-\delta \varphi i_{\xi} E_{\varphi} \\
& +i_{\xi} e \cdot \delta E_{e}+\left(i_{\xi} \omega-\chi_{\xi}\right) \cdot \delta E_{\omega}+i_{\xi} h \cdot \delta E_{h} \\
& +\delta \Theta\left(\Phi, \delta_{\xi} \Phi\right)-\delta_{\xi} \Theta(\Phi, \delta \Phi)
\end{aligned}
$$

and we will refer to that as "the generalized off-shell ADT current" $[30,31]$. If $\xi$ is a Killing vector field everywhere, the generalized off-shell ADT current is reduced to the ordinary one because we have the following configuration space result: [53],

$\delta \Theta\left(\Phi, \delta_{\xi} \Phi\right)-\delta_{\xi} \Theta(\Phi, \delta \Phi)=0 ;$ 
this equality holds when $\xi$ is a Killing vector field. Also, if the equations of motion and the linearized equations of motion both are satisfied, the off-shell ADT current is reduced to the symplectic current. We know that the symplectic current gives conserved charges associated with asymptotically Killing vector fields [25]. Thus this generalization makes sense for an ordinary ADT current. In this way, the generalized off-shell ADT current will be conserved for any asymptotically Killing vector field as well as for a Killing vector field which is admitted everywhere by spacetime. It seems that we can write $[29,42]$,

$\delta \psi_{\xi}-\Pi_{\xi}=\mathrm{d} Z_{\xi}$,

so Eq. (55) can be rewritten as

$\mathfrak{J}_{\mathrm{ADT}}(\Phi, \delta \Phi ; \xi)=\mathrm{d} \mathfrak{Q}_{\mathrm{ADT}}(\Phi, \delta \Phi ; \xi)$

where $\mathfrak{Q}_{\mathrm{ADT}}(\Phi, \delta \Phi ; \xi)$ is a generalized off-shell ADT conserved charge associated with an asymptotically Killing vector field $\xi$, which is given by

$\mathfrak{Q}_{\mathrm{ADT}}(\Phi, \delta \Phi ; \xi)=\delta K_{\xi}-i_{\xi} \Theta(\Phi, \delta \Phi)+Z_{\xi}$.

The quasi-local conserved charge associated with the Killing vector field can be defined by $\xi$ as $[28,29]$,

$Q(\xi)=\frac{1}{8 \pi G} \int_{0}^{1} \mathrm{~d} s \int_{\Sigma} \mathfrak{Q}_{\mathrm{ADT}}(\Phi \mid s)$

where $G$ denotes the Newtonian gravitational constant and $\Sigma$ is a space-like codimension two surface. Also, integration over $s$ is just integration over an one-parameter path in the solution space and $s=0$ and $s=1$ correspond to the background solution and the solution of interest, respectively.

It is straightforward to calculate $\psi_{\xi}$ in Eq. (46) using the fact that the exterior derivative and the $\mathrm{L}-\mathrm{L}$ derivative do not commute,

$\left[d, \mathfrak{L}_{\xi}\right] e=\mathrm{d} \chi_{\xi} \times e$.

Thus we find

$\psi_{\xi}=\mathrm{d} \chi_{\xi} \cdot\left[-\frac{1}{2} \varphi e+\frac{1}{2 \mu} \omega\right]$.

In a similar way, $\Pi_{\xi}$ can be obtained in Eq. (54)

$$
\Pi_{\xi}=\mathrm{d} \chi_{\xi} \cdot\left[-\frac{1}{2} \delta \varphi e-\frac{1}{2} \varphi \delta e+\frac{1}{2 \mu} \delta \omega\right] .
$$

It is easy to see from Eqs. (58), (63) and (64) that $d Z_{\xi}=0$. We then choose $Z_{\xi}$ to be zero. As mentioned earlier, $J_{\xi}=$ $\mathrm{d} K_{\xi}$ can be written by the Poincaré lemma. So, from Eq. (53), $K_{\xi}$ is found as follows:

$$
\begin{aligned}
K_{\xi}= & \varphi\left(i_{\xi} \omega-\chi_{\xi}\right) \cdot e+\frac{1}{2 \mu} i_{\xi} \omega \cdot \omega-\frac{1}{\mu} \chi \xi \cdot \omega+\frac{1}{2} \varphi^{2} i_{\xi} e \cdot e \\
& +i_{\xi} e \cdot h+\frac{1}{2} \varphi i_{\xi} \omega \cdot e-\frac{1}{2} \varphi i_{\xi} e \cdot \omega .
\end{aligned}
$$

Considering the above results, namely Eqs. (63)-(65) and taking into account Eq. (24), the generalized ADT conserved charge (60) can be calculated

$$
\begin{aligned}
& \mathfrak{Q}_{\mathrm{ADT}}(\Phi, \delta \Phi ; \xi) \\
& \quad=\left[\left(i_{\xi} \Omega-\chi_{\xi}\right) \cdot e-\alpha i_{\xi} h \cdot e+\varphi i_{\xi} e \cdot e\right] \delta \varphi \\
& \quad+\left[\varphi\left(i_{\xi} \Omega-\chi_{\xi}\right)+\varphi^{2} i_{\xi} e+(1-\alpha \varphi) i_{\xi} h\right] \cdot \delta e \\
& \quad+\left[\varphi i_{\xi} e+\frac{1}{\mu}\left(i_{\xi} \Omega-\chi_{\xi}\right)-\frac{\alpha}{\mu} i_{\xi} h\right] \cdot \delta \Omega \\
& \quad+\left[(1-\alpha \varphi) i_{\xi} e-\frac{\alpha}{\mu}\left(i_{\xi} \Omega-\chi_{\xi}\right)+\frac{\alpha^{2}}{\mu} i_{\xi} h\right] \cdot \delta h .
\end{aligned}
$$

By explicitly demanding that $\delta_{\xi} e^{a}=0$ when $\xi$ is a Killing vector field, we find the following expression for $\chi \xi[50,52]$ :

$\chi_{\xi}^{a}=i_{\xi} \omega^{a}+\frac{1}{2} \varepsilon^{a}{ }_{b c} e^{\nu b}\left(i_{\xi} T^{c}\right)_{\nu}+\frac{1}{2} \varepsilon^{a}{ }_{b c} e^{b}{ }_{\mu} e^{c}{ }_{\nu} \nabla^{\mu} \xi^{\nu}$.

Now, this expression is simplified for the considered model, which is not torsion free. It is clear that the covariant derivative of a vector field $\xi$ is given by

$\nabla_{\mu} \xi^{v}=\partial_{\mu} \xi^{v}+\Gamma_{\mu \sigma}^{v} \xi^{\sigma}$

where $\Gamma_{\mu \nu}^{\alpha}$ is the connection compatible with metric $g_{\mu \nu}$. On the one hand, we can decompose this connection into two parts

$\Gamma_{\mu \nu}^{\alpha}=\left\{\begin{array}{c}\alpha \\ \mu \nu\end{array}\right\}+K_{\mu \nu}^{\alpha}$

where $\left\{\mu_{\mu \nu}^{\alpha}\right\}$ is the Levi-Civita connection and $K_{\mu \nu}^{\alpha}$ is the contorsion tensor, which is defined by

$K_{\mu \nu}^{\alpha}=T_{\mu \nu}^{\alpha}+T_{\mu}^{\alpha}{ }_{\nu}+T_{\nu}{ }^{\alpha}{ }_{\mu}$

in terms of Cartan torsion tensor $T_{\mu \nu}^{\alpha}=\frac{1}{2}\left(\Gamma_{\mu \nu}^{\alpha}-\Gamma_{\nu \mu}^{\alpha}\right)$. Thus we have

$K_{\mu \nu}^{\alpha}=2 T_{\mu \nu}^{\alpha}-\Gamma_{\mu \nu}^{\alpha}$.

Now, by substituting Eqs. (69) and (71) into Eq. (67) we will find the following expression for $\chi_{\xi}$ :

$\chi_{\xi}^{a}=i_{\xi} \Omega^{a}+\frac{1}{2} \varepsilon^{a}{ }_{b c} e^{b}{ }_{\mu} e^{c}{ }_{\nu} \tilde{\nabla}^{\mu} \xi^{\nu}$, 
where $\tilde{\nabla}$ denotes the covariant derivative with respect to the Levi-Civita connection. Hence, to calculate the conserved charges of the solutions considered by using Eq. (61), the above expression can be employed for $\chi \xi$. In the above procedure to find conserved charges we assume that $\varphi$ as well as $e$ and $\omega$ is a dynamical field which is an arbitrary function of coordinates. So it is clear that Eqs. (61), (66) and (72) can be used to obtain charges associated with the solutions of the theory, which may have a non-constant scalar field.

\section{General formula for entropy of black holes in minimal massive gravity coupled to scalar field}

When we are considering a stationary black hole solution of the minimal massive gravity coupled to a scalar field we know that the entropy of a black hole is a conserved charge associated with the Killing horizon generating Killing field $\zeta$ [25]. We take the codimension two surface $\Sigma$ to be the bifurcation surface $\mathcal{B}$. Assuming that $\zeta$ is the Killing vector field which generates the Killing horizon, we must set $\zeta=0$ on $\mathcal{B}$. Thus Eq. (66) becomes

$\mathfrak{Q}_{\mathrm{ADT}}(\Phi, \delta \Phi ; \zeta)=-\delta\left[\chi_{\zeta} \cdot\left(\varphi e+\frac{1}{\mu} \Omega-\frac{\alpha}{\mu} h\right)\right]$

on the bifurcation surface. Now take $s=0$ and $s=1$ corresponding to the considered black hole spacetime and the perturbed one, respectively. Therefore, by integrating (73) over the one-parameter path in the solution space we find

$$
\int_{0}^{1} \mathrm{~d} s \mathfrak{Q}_{\mathrm{ADT}}(\Phi, \delta \Phi ; \zeta)=-\chi_{\zeta} \cdot\left[\varphi e+\frac{1}{\mu} \Omega-\frac{\alpha}{\mu} h\right] .
$$

The bifurcation surface of a stationary black hole is a circle. In Ref. [50] the authors have shown that on the bifurcation surface we can write

$\chi_{\zeta}^{a}=\kappa N^{a}$

where $\kappa$ is the surface gravity and $N^{a}$ is a vector which is given by

$N^{\mu}=\left(0,0, \frac{1}{\sqrt{g_{\phi \phi}}}\right)$.

Hence, by substituting Eq. (74) into Eq. (61) we will find

$Q(\zeta)=-\frac{\kappa}{8 \pi G} \int_{0}^{2 \pi} \frac{\mathrm{d} \phi}{\sqrt{g_{\phi \phi}}}\left[\varphi g_{\phi \phi}+\frac{1}{\mu} \Omega_{\phi \phi}-\frac{\alpha}{\mu} h_{\phi \phi}\right]$, which should be calculated on the horizon. Now, the entropy of a stationary black hole can be defined [25]

$S=-\frac{2 \pi}{\kappa} Q(\zeta)$

therefore,

$S=\frac{1}{4 G} \int_{0}^{2 \pi} \frac{\mathrm{d} \phi}{\sqrt{g_{\phi \phi}}}\left[\varphi g_{\phi \phi}+\frac{1}{\mu} \Omega_{\phi \phi}-\frac{\alpha}{\mu} h_{\phi \phi}\right]$,

which should be calculated on the horizon. In the above formula, $h_{\phi \phi}$ is given by

$$
\begin{aligned}
h_{\phi \phi}= & -\frac{1}{\left[\mu(1-\alpha \varphi)^{2}-\alpha^{2} \varphi^{2}\right]} \\
& \times\left\{S_{\phi \phi}+\frac{1}{2}[\alpha \lambda \varphi+\mu(1-\alpha \varphi)] \varphi^{2} g_{\phi \phi}\right\} .
\end{aligned}
$$

Equation (79) will be similar to the ordinary minimal massive gravity formula when we take $\varphi=\varphi_{0}$.

\section{Application for BTZ black hole solution with $\varphi=\varphi_{0}$}

In this section, the conserved charges and entropy of the BTZ black hole solution with $\varphi=\varphi_{0}$ in the context of the considered model is calculated. The following dreibein describes the BTZ black hole spacetime:

$e^{0}=\left(\frac{\left(r^{2}-r_{+}^{2}\right)\left(r^{2}-r_{-}^{2}\right)}{l^{2} r^{2}}\right)^{\frac{1}{2}} \mathrm{~d} t$

$e^{1}=\left(\frac{l^{2} r^{2}}{\left(r^{2}-r_{+}^{2}\right)\left(r^{2}-r_{-}^{2}\right)}\right)^{\frac{1}{2}} \mathrm{~d} r$

$e^{2}=r\left(\mathrm{~d} \phi-\frac{r_{+} r_{-}}{l r^{2}} \mathrm{~d} t\right)$,

where $r_{+}$and $r_{-}$are the outer and inner horizon radii of the BTZ black hole, respectively. We take the integration surface $\Sigma$ to be a circle with an infinite radius. Therefore, we can consider the $\mathrm{AdS}_{3}$ spacetime to be a background corresponding to $s=0$,

$\bar{e}^{0}=\frac{r}{l} \mathrm{~d} t, \quad \bar{e}^{-1}=\frac{l}{r} \mathrm{~d} r, \quad \bar{e}^{2}=r \mathrm{~d} \phi$.

The bar on a quantity means that the quantity is calculated on the background. Thus Eq. (66) for the considered solution becomes 


$$
\begin{aligned}
\mathfrak{Q}_{\mathrm{ADT}}(\Phi, \delta \Phi ; \xi)= & {\left[\left(\varphi_{0}-\frac{\alpha \beta}{\mu}\right)\left(i_{\xi} \bar{\Omega}-\bar{\chi}_{\xi}\right)+\frac{1}{\mu l^{2}} i_{\xi} \bar{e}\right] \cdot \delta e } \\
& +\left[\left(\varphi_{0}-\frac{\alpha \beta}{\mu}\right) i_{\xi} \bar{e}+\frac{1}{\mu}\left(i_{\xi} \bar{\Omega}-\bar{\chi}_{\xi}\right)\right] \cdot \delta \Omega
\end{aligned}
$$

where we have used Eq. (38). Quantities that are calculated on the background do not obviously depend on parameters of the solution space, so by taking an integration from Eq. (83) over a one-parameter path in the solution space, we find

$$
\begin{aligned}
\int_{0}^{1} \mathrm{~d} s \mathfrak{Q}_{\mathrm{ADT}}(\Phi, \delta \Phi ; \xi)= & {\left[\left(\varphi_{0}-\frac{\alpha \beta}{\mu}\right)\left(i_{\xi} \bar{\Omega}-\bar{\chi}_{\xi}\right)\right.} \\
& \left.+\frac{1}{\mu l^{2}} i_{\xi} \bar{e}\right] \cdot \Delta e \\
& +\left[\left(\varphi_{0}-\frac{\alpha \beta}{\mu}\right) i_{\xi} \bar{e}\right. \\
& \left.+\frac{1}{\mu}\left(i_{\xi} \bar{\Omega}-\bar{\chi}_{\xi}\right)\right] \cdot \Delta \Omega,
\end{aligned}
$$

where $\Delta \Phi=\Phi_{(s=1)}-\Phi_{(s=0)}$. By substituting Eq. (84) into Eq. (61) we find that

$$
\begin{aligned}
Q(\xi)= & \frac{1}{8 \pi G} \lim _{r \rightarrow \infty} \int_{0}^{2 \pi}\left\{\left[\left(\varphi_{0}-\frac{\alpha \beta}{\mu}\right)\left(i_{\xi} \bar{\Omega}-\bar{\chi}_{\xi}\right)\right.\right. \\
& \left.+\frac{1}{\mu l^{2}} i_{\xi} \bar{e}\right] \cdot \Delta e_{\phi} \\
& +\left[\left(\varphi_{0}-\frac{\alpha \beta}{\mu}\right) i_{\xi} \bar{e}\right. \\
& \left.\left.+\frac{1}{\mu}\left(i_{\xi} \bar{\Omega}-\bar{\chi}_{\xi}\right)\right] \cdot \Delta \Omega_{\phi}\right\} \mathrm{d} \phi .
\end{aligned}
$$

For a BTZ black hole spacetime at spatial infinity we have

$$
\begin{aligned}
\Delta e^{a}{ }_{\phi} & =0, \quad \Delta \Omega_{\phi}^{\hat{t}}=-\frac{r_{+}^{2}+r_{-}^{2}}{2 l r}, \\
\Delta \Omega^{\hat{r}}{ }_{\phi} & =0, \quad \Delta \Omega_{\phi}^{\hat{\phi}}=-\frac{r_{+} r_{-}}{l r},
\end{aligned}
$$

which are calculated based on Eq. (81).

Energy is a conserved charge corresponding to the Killing vector $\xi=\partial_{t}$, thus

$E=\frac{1}{8 G}\left[\left(\varphi_{0}-\frac{\alpha \beta}{\mu}\right)\left(\frac{r_{+}^{2}+r_{-}^{2}}{l^{2}}\right)-\frac{2 r_{+} r_{-}}{\mu l^{3}}\right]$.

For the given solution we have $\alpha \beta=0$ (see Eq. (38)), thus the contribution from the MMG vanishes and the expression for the energy (88) becomes

$E=\frac{1}{8 G}\left[\varphi_{0}\left(\frac{r_{+}^{2}+r_{-}^{2}}{l^{2}}\right)-\frac{2 r_{+} r_{-}}{\mu l^{3}}\right]$
The angular momentum is a conserved charge corresponding to the Killing vector $\xi=-\partial_{\phi}$. Then

$j=\frac{1}{8 G}\left[\left(\varphi_{0}-\frac{\alpha \beta}{\mu}\right)\left(\frac{2 r_{+} r_{-}}{l}\right)-\frac{r_{+}^{2}+r_{-}^{2}}{\mu l^{2}}\right]$.

Similarly, due to Eq. (38), the expression for the angular momentum can be written

$j=\frac{1}{8 G}\left[\varphi_{0}\left(\frac{2 r_{+} r_{-}}{l}\right)-\frac{r_{+}^{2}+r_{-}^{2}}{\mu l^{2}}\right]$.

Since on the horizon of the BTZ black hole we find

$g_{\phi \phi}=r_{+}^{2}, \quad \Omega_{\phi \phi}=-\frac{r_{+} r_{-}}{l}, \quad h_{\phi \phi}=\beta r_{+}^{2}$,

by substituting Eq. (91) into Eq. (79) we find the entropy of the BTZ black hole solution,

$S=\frac{\pi}{2 G}\left[\left(\varphi_{0}-\frac{\alpha \beta}{\mu}\right) r_{+}-\frac{r_{-}}{\mu l}\right]$.

Again, because of Eq. (38), the contribution from the MMG term in the entropy will vanish, i.e.

$S=\frac{\pi}{2 G}\left[\varphi_{0} r_{+}-\frac{r_{-}}{\mu l}\right]$.

It is straightforward to check that these results satisfy the first law of black hole thermodynamics, that is,

$\delta E=T_{H} \delta S+\Omega_{H} \delta j$,

where $T_{H}=\frac{\kappa}{2 \pi}$ and $\Omega_{H}=\frac{r_{-}}{l r_{+}}$are the Hawking temperature and the angular velocity of the horizon, respectively.

\section{Virasoro algebra and the central term}

Using the results of Sect. 4 , the central extension term for the considered model can be obtained and subsequently the central charges can be read. In this section, we take $\mathrm{AdS}_{3}$ spacetime with $\varphi=\varphi_{0}$ as background (see Eq. (82)) and the integration surface $\Sigma$ to be a circle with a radius of infinity. Two copies of the classical centerless Virasoro algebra, which are known as the Witt algebra, are given by,

$\left[\xi_{m}^{ \pm}, \xi_{n}^{ \pm}\right]=i(n-m) \xi_{m+n}^{ \pm}, \quad\left[\xi_{m}^{+}, \xi_{n}^{-}\right]=0$,

where $\xi_{m}^{ \pm}(m \in \mathbb{Z})$ are the vector fields. They have the following form [54]:

$\xi_{n}^{ \pm}=\frac{1}{2} e^{i n x^{ \pm}}\left[l\left(1-\frac{l^{2} n^{2}}{2 r^{2}}\right) \partial_{t}-i n r \partial_{r} \pm\left(1+\frac{l^{2} n^{2}}{2 r^{2}}\right) \partial_{\phi}\right]$, 
where $x^{ \pm}=t / l \pm \phi$. The square brackets in Eq. (95) denote the Lie bracket. The central extension term $C\left(\xi_{m}^{ \pm}, \xi_{n}^{ \pm}\right)$is given by the following Eq. [55] (see also related work in $[56,57])$ :

$\left\{Q\left(\xi_{m}^{ \pm}\right), Q\left(\xi_{n}^{ \pm}\right)\right\}=Q\left(\left[\xi_{m}^{ \pm}, \xi_{n}^{ \pm}\right]\right)+C\left(\xi_{m}^{ \pm}, \xi_{n}^{ \pm}\right)$.

Since the conserved charge (61) is linear in $\xi$,

$Q\left(\left[\xi_{m}^{ \pm}, \xi_{n}^{ \pm}\right]\right)=i(n-m) Q\left(\xi_{m+n}^{ \pm}\right)$

on the other hand, we know that

$\left\{Q\left(\xi_{m}^{ \pm}\right), Q\left(\xi_{n}^{ \pm}\right)\right\}=\delta_{\xi_{n}^{ \pm}} Q\left(\xi_{m}^{ \pm}\right)$.

Thus the central extension term will be obtained from the following equation:

$C\left(\xi_{m}^{ \pm}, \xi_{n}^{ \pm}\right)=\delta_{\xi_{n}^{ \pm}} Q\left(\xi_{m}^{ \pm}\right)-i(n-m) Q\left(\xi_{m+n}^{ \pm}\right)$.

It is clear from Eq. (61) that we can write at spatial infinity

$\delta Q(\xi)=\frac{1}{8 \pi G} \int_{\infty} \mathfrak{Q}_{\mathrm{ADT}}(\bar{\Phi}, \delta \Phi ; \xi)$

Therefore, by taking an integration from Eq. (101) over an one-parameter path in the solution space, we find

$Q(\xi)=\frac{1}{8 \pi G} \int_{\infty} \mathfrak{Q}_{\mathrm{ADT}}(\bar{\Phi}, \Delta \Phi ; \xi)$.

Also, from Eq. (101), it can easily be deduced that

$\delta_{\xi_{n}^{ \pm}} Q\left(\xi_{m}^{ \pm}\right)=\frac{1}{8 \pi G} \int_{\infty} \mathfrak{Q}_{\mathrm{ADT}}\left(\bar{\Phi}, \delta_{\xi_{n}^{ \pm}} \Phi ; \xi_{m}^{ \pm}\right)$

Thus, by substituting Eqs. (102) and (103) into (100), an expression for the central extension term is found. Consequently the central charges of the considered model can be read. Because we take $\mathrm{AdS}_{3}$ spacetime with $\varphi=\varphi_{0}$ then Eq. (103) can be rewritten as

$$
\begin{aligned}
\delta_{\xi_{n}^{ \pm}} Q\left(\xi_{m}^{ \pm}\right)= & \frac{1}{8 \pi G}\left(\varphi_{0}-\frac{\alpha \beta}{\mu} \pm \frac{1}{\mu l}\right) \lim _{r \rightarrow \infty} \\
& \times \int_{0}^{2 \pi} i_{\xi_{m}^{ \pm}} \bar{e} \cdot \delta_{\xi_{n}^{ \pm}} A_{\phi}^{ \pm} \mathrm{d} \phi,
\end{aligned}
$$

where $A^{ \pm}$are connections corresponding to the two $S O(2,1)$ gauge groups [58]

$\left(A^{ \pm}\right)^{a}=\Omega^{a} \pm \frac{1}{l} e^{a}$.
In the calculation of Eq. (104) we have used the following equation:

$i_{\xi_{n}^{ \pm}} \bar{\Omega}-\bar{\chi}_{\xi_{n}^{ \pm}}= \pm \frac{1}{l} i_{\xi_{n}^{ \pm}} \bar{e}$,

which can be derived from Eqs. (72) and (96). By using Eqs. (24) and (29), Eqs. (47)-(50) can be simplified as follows:

$$
\begin{aligned}
\delta_{\xi} e & =D(\Omega) i_{\xi} e+\left(\chi_{\xi}-i_{\xi} \Omega\right) \times e, \\
\delta_{\xi} \Omega & =i_{\xi} R(\Omega)+D(\Omega)\left(i_{\xi} \Omega-\chi \xi\right), \\
\delta_{\xi} h & =D(\Omega) i_{\xi} h+i_{\xi} D(\Omega) h+\left(\chi \xi-i_{\xi} \Omega\right) \times h, \\
\delta_{\xi} \varphi & =i_{\xi} D(\Omega) \varphi,
\end{aligned}
$$

therefore, it can be shown that

$$
\begin{aligned}
& \delta_{\xi_{n}^{ \pm}}\left(A^{ \pm}\right)_{\phi}^{0}=-\frac{i \ln ^{3}}{2 r} e^{i n x^{ \pm}}, \\
& \delta_{\xi_{n}^{ \pm}}\left(A^{ \pm}\right)_{\phi}^{1}=0 \text {, } \\
& \delta_{\xi_{n}^{ \pm}}\left(A^{ \pm}\right)_{\phi}^{2}= \pm \frac{i l n^{3}}{2 r} e^{i n x^{ \pm}} \text {. }
\end{aligned}
$$

By substituting Eq. (111) into Eq. (104), we find that

$\delta_{\xi_{n}^{ \pm}} Q\left(\xi_{m}^{ \pm}\right)=\frac{i l^{3}}{8 G}\left(\varphi_{0}-\frac{\alpha \beta}{\mu} \pm \frac{1}{\mu l}\right) \delta_{m+n, 0}$

Suppose that $\varphi=\varphi_{0}$ and $h=\beta e$, as they are sensible for the BTZ black hole solution. Then Eq. (102) for $\xi=\xi_{m}^{ \pm}$ becomes

$Q\left(\xi_{m}^{ \pm}\right)=\frac{1}{8 \pi G}\left(\varphi_{0}-\frac{\alpha \beta}{\mu} \pm \frac{1}{\mu l}\right) \lim _{r \rightarrow \infty} \int_{0}^{2 \pi} i_{\xi_{m}^{ \pm}} \bar{e} \cdot \Delta A_{\phi}^{ \pm} \mathrm{d} \phi$

By substituting Eq. (86) into (113), we find that

$$
Q\left(\xi_{m}^{ \pm}\right)=\frac{l}{16 G}\left(\varphi_{0}-\frac{\alpha \beta}{\mu} \pm \frac{1}{\mu l}\right)\left(\frac{r_{+} \mp r_{-}}{l}\right)^{2} \delta_{m, 0}
$$

Now, to find the central extension term we must substitute Eqs. (112) and (114) into (100). We find that

$$
\begin{aligned}
C\left(\xi_{m}^{ \pm}, \xi_{n}^{ \pm}\right)= & \frac{i l}{8 G}\left(\varphi_{0}-\frac{\alpha \beta}{\mu} \pm \frac{1}{\mu l}\right) \\
& \times\left[n^{3}-\left(\frac{r_{+} \mp r_{-}}{l}\right)^{2} n\right] \delta_{m+n, 0} .
\end{aligned}
$$

To obtain the usual $n$ dependence, that is, $n\left(n^{2}-1\right)$, in the above expression it is sufficient that a shift is made from $Q\left(\xi_{m}^{ \pm}\right)$by a constant [59]. Thus by the substitution 
$Q\left(\xi_{n}^{ \pm}\right) \equiv L_{n}^{ \pm}, \quad\left\{Q\left(\xi_{m}^{ \pm}\right), Q\left(\xi_{n}^{ \pm}\right)\right\} \equiv i\left[L_{m}^{ \pm}, L_{n}^{ \pm}\right]$,

Equation (100) becomes

$\left[L_{m}^{ \pm}, L_{n}^{ \pm}\right]=(n-m) L_{m+n}^{ \pm}+\frac{c_{ \pm}}{12} n\left(n^{2}-1\right) \delta_{m+n, 0}$,

where

$c_{ \pm}=\frac{3 l}{2 G}\left(\varphi_{0}-\frac{\alpha \beta}{\mu} \pm \frac{1}{\mu l}\right)$

are central charges and $L_{n}^{ \pm}$are generators of Virasoro algebra. So the algebra among the conserved charges is isomorphic to two copies of the Virasoro algebra. The eigenvalues of the Virasoro generators $L_{n}^{ \pm}$can be read off Eq. (114),

$l_{n}^{ \pm}=\frac{l}{16 G}\left(\varphi_{0}-\frac{\alpha \beta}{\mu} \pm \frac{1}{\mu l}\right)\left(\frac{r_{+} \mp r_{-}}{l}\right)^{2} \delta_{m, 0}$.

By virtue of Eq. (38) the contribution from the MMG in the central charges and eigenvalues of the Virasoro generators will vanish, so we find

$c_{ \pm}=\frac{3 l}{2 G}\left(\varphi_{0} \pm \frac{1}{\mu l}\right)$,

$l_{n}^{ \pm}=\frac{l}{16 G}\left(\varphi_{0} \pm \frac{1}{\mu l}\right)\left(\frac{r_{+} \mp r_{-}}{l}\right)^{2} \delta_{m, 0}$,

for cental charges and eigenvalues of the Virasoro generators, respectively. The eigenvalues of the Virasoro generators $L_{n}^{ \pm}$ are related to the energy $E$ and the angular momentum $j$ of the BTZ black hole by the following equations, respectively:

$$
\begin{gathered}
E=\frac{1}{l}\left(l_{0}^{+}+l_{0}^{-}\right)=\frac{1}{8 G}\left[\varphi_{0}\left(\frac{r_{+}^{2}+r_{-}^{2}}{l^{2}}\right)-\frac{2 r_{+} r_{-}}{\mu l^{3}}\right], \\
j=l_{0}^{-}-l_{0}^{+}=\frac{1}{8 G}\left[\varphi_{0}\left(\frac{2 r_{+} r_{-}}{l}\right)-\frac{r_{+}^{2}+r_{-}^{2}}{\mu l^{2}}\right] .
\end{gathered}
$$

Also, to calculate the entropy of the black hole considered the Cardy formula can be used [60,61] (see also [54])

$S=2 \pi \sqrt{\frac{c_{+} L_{0}^{+}}{6}}+2 \pi \sqrt{\frac{c_{-} L_{0}^{-}}{6}}=\frac{\pi}{2 G}\left[\varphi_{0} r_{+}-\frac{r_{-}}{\mu l}\right]$.

By comparing the above results, Eqs. (122)-(124), with Eqs. (88), (90) and (93) it can be seen that they match exactly.

\section{Conclusion}

In this paper, the theory of topologically massive gravity non-minimally coupled to a scalar field which comes from the Lorentz-Chern-Simons theory [1] is considered. The
Lagrangian of this theory is given by Eq. (9). It is a torsionfree one. The theory is extended by adding an extra term (13) which makes the torsion non-zero. The Lagrangian of the extended theory is given by Eq. (14). In Sect. 3, we have obtained the equations of motion (25)-(28) of the extended theory in such a way that they are expressed in terms of the usual torsion-free spin connection (24). We have shown that the BTZ spacetime together with $\varphi=\varphi_{0}$ solves the equations of motion (25)-(28) when one of Eq. (40) or (41) is satisfied. In Sect. 4, the off-shell ADT current is generalized and it is deduced that it is conserved for any asymptotically Killing vector field as well as a Killing vector field which is admitted by spacetime everywhere. Then the Poincaré lemma is used to define the generalized off-shell ADT charge (60). Consequently, we have defined the quasi-local conserved charge (61) for the considered theory. In Sect. 5 a general formula (79) is found for the entropy of the stationary black hole solution in the context of the considered theory. We have used the obtained formulas to calculate the energy (88), the angular momentum (90) and the entropy (93) of the BTZ black hole solution. These quantities satisfy the first law of black hole mechanics. In Sect. 7 we have obtained the central extension term (115) and then read off the central charges (120) and the eigenvalues of the Virasoro algebra generators (121) for the BTZ black hole solution. We have calculated the energy (122) and the angular momentum (123) of this black hole using the eigenvalues of the Virasoro algebra generators. Also, we have calculated the entropy of the BTZ black hole by using the Cardy formula (124). Comparing Eqs. (122)-(124) with Eqs. (88), (90) and (93) we found that, although they have been obtained in two different ways, they exactly match, as we expected.

We should mention that here we have considered a special example, a BTZ black hole together with $\varphi=\varphi_{0}$ where $\varphi_{0}$ is a constant. In this case, the equations of motion (26) and (27) imply that $h^{a}$ is proportional to $e^{a}$ (see Eq. (33)). Thus, on one hand, the contributions from the MMG interaction term in the energy, the angular momentum and the entropy become proportional to $\alpha \beta$ (see Eqs. (66), (79)). On the other hand, the remaining equation of motion (25) requires that $\alpha \beta=$ 0 . Therefore, the contributions from the MMG interaction term in the energy, angular momentum and entropy vanish for the given example. In the model considered, we have one more equation of motion than in the ordinary $\mathrm{MMG}$ model. Herewith we can deduce that the coupling to the scalar field removes the dependence on $\alpha$ for the given example. A similar argument holds for the Virasoro central charges.

We do not expect that the results obtained match with the MMG ones when we take a constant scalar limit. The reason for this comes from the fact that the Lagrangian (14) does not become the Lagrangian of the MMG [9] when the scalar field is a constant. Instead, it will reduce to the Lagrangian of the Mielke-Baekler model with torsion when the scalar field 
is considered as a constant (the Mielke-Baekler Lagrangian (15) has an extra term $\theta_{T} e \cdot T(\omega)$ over the TMG. Subsequently, the Lagrangian (14), when the scalar field is a constant, differs from the Lagrangian of the MMG by a term of the form $\left.\theta_{T} e \cdot T(\omega)\right)$.

Acknowledgements M. R. Setare thanks Simon del Pino and Gaston Giribet for helpful comments.

Open Access This article is distributed under the terms of the Creative Commons Attribution 4.0 International License (http://creativecomm ons.org/licenses/by/4.0/), which permits unrestricted use, distribution, and reproduction in any medium, provided you give appropriate credit to the original author(s) and the source, provide a link to the Creative Commons license, and indicate if changes were made.

Funded by SCOAP ${ }^{3}$.

\section{References}

1. S. del Pino, G. Giribet, A. Toloza, J. Zanelli, JHEP 06, 113 (2015)

2. L.F. Abbott, S. Deser, Nucl. Phys. B 195, 76 (1982)

3. L.F. Abbott, S. Deser, Phys. Lett. B 116, 259 (1982)

4. S. Deser, B. Tekin, Phys. Rev. D 67, 084009 (2003)

5. S. Deser, B. Tekin, Phys. Rev. Lett. 89, 101101 (2002)

6. S. Deser, R. Jackiw, S. Templeton, Ann. Phys. 140, 372 (1982) [Erratum-ibid. 185, 406.1988 APNYA, 281,409 (1988 APNYA,281,409-449.2000)]

7. K.A. Moussa, G. Clement, C. Leygnac, Class. Quantum Grav. 20, L277 (2003)

8. S. Deser, B. Tekin, Class. Quantum Grav. 20, L259 (2003)

9. E. Bergshoeff, O. Hohm, W. Merbis, A.J. Routh, P.K. Townsend, Class. Quantum Grav. 31, 145008 (2014)

10. A.S. Arvanitakis, A.J. Routh, P.K. Townsend, Class. Quantum Grav. 31, 235012 (2014)

11. A. Baykal, Class. Quantum Grav. 32, 025013 (2015)

12. A.S. Arvanitakis, P.K. Townsend, Class. Quantum Grav. 32, $085003(2015)$

13. M. Alishahiha, M.M. Qaemmaqami, A. Naseh, A. Shirzad. arXiv:1409.6146 [hep-th]

14. G. Giribet, Y. Vsquez, Phys. Rev. D 91, 024026 (2015)

15. M.R. Setare, Nucl. Phys. B 898, 259 (2015)

16. M.R. Setare, H. Adami, Phys. Rev. D 91, 104039 (2015)

17. A.S. Arvanitakis, Class. Quantum Grav. 32, 115010 (2015)

18. M.R. Setare, H. Adami, Phys. Lett. B 744, 280 (2015)

19. W. Li, W. Song, A. Strominger, JHEP 0804, 082 (2008)

20. M. Henneaux, C. Martinez, R. Troncoso, J. Zanelli, Phys. Rev. D 65, 104007 (2002)
21. G. de Berredo-Peixoto, Class. Quantum Grav. 20, 3983 (2003)

22. J. Gegenberg, C. Martinez, R. Troncoso, Phys. Rev. D 67, 084007 (2003)

23. F. Correa, A. Faundez, C. Martinez, Phys. Rev. D 87, 027502 (2013)

24. B. Tekin, Phys. Rev. D 90, 081701 (2014)

25. R.M. Wald, Phys. Rev. D 48, 3427 (1993)

26. R.L. Arnowitt, S. Deser, C.W. Misner, Gen. Rel. Grav. 40, 1997 (2008)

27. J.D. Brown, J.W. York, Phys. Rev. D 47, 1407 (1993)

28. W. Kim, S. Kulkarni, S.H. Yi, Phys. Rev. Lett. 111, 081101 (2013)

29. W. Kim, S. Kulkarni, S.H. Yi, Phys. Rev. D 88, 124004 (2013)

30. S. Hyun, S.A. Park, S.H. Yi, JHEP 06, 151 (2014)

31. M.R. Setare, H. Adami, Nucl. Phys. B 909, 345 (2016)

32. H. Adami, M.R. Setare, Eur. Phys. J. C 76, 187 (2016)

33. A. Bouchareb, G. Clement, Class. Quantum Grav. 24, 5581 (2007)

34. S. Hyun, J. Jeong, S.A. Park, S.H. Yi, Phys. Rev. D 90, 104016 (2014)

35. G. Clement, Phys. Rev. D 49, 5131 (1994)

36. G. Clement, Class. Quantum Grav. 11, L115 (1994)

37. O. Miskovic, R. Olea, JHEP 0912, 046 (2009)

38. G. Giribet, M. Leston, JHEP 1009, 070 (2010)

39. O. Hohm, E. Tonni, JHEP 1004, 093 (2010)

40. S. Nam, J.D. Park, S.H. Yi, JHEP 1007, 058 (2010)

41. S. Nam, J.D. Park, S.H. Yi, Phys. Rev. D 82, 124049 (2010)

42. Y. Tachikawa, Class. Quantum Grav. 24, 737 (2007)

43. L. Bonora, M. Cvitan, P. Dominis Prester, S. Pallua, I. Smolic, JHEP 1107, 085 (2011)

44. T. Azeyanagi, R. Loganayagam, G.S. Ng, M.J. Rodriguez. arXiv:1407.6364 [hep-th]

45. E.A. Bergshoeff, O. Hohm, W. Merbis, A.J. Routh, P.K. Townsend, Lect. Notes Phys. 892, 181 (2015)

46. E.W. Mielke, P. Baekler, Phys. Lett. A 156, 399 (1991)

47. M. Blagojevic, B. Cvetkovic, Class. Quantum Grav. 23, 4781 (2006)

48. M. Blagojevic, B. Cvetkovic, JHEP 0610, 005 (2006)

49. M. Blagojevic, B. Cvetkovic, Phys. Rev. D 78, 044036 (2008)

50. M.R. Setare, H. Adami, Nucl. Phys. B 902, 115 (2016)

51. M. Banados, C. Teitelboim, J. Zanelli, Phys. Rev. Lett. 69, 1849 (1992)

52. T. Jacobson, A. Mohd, Phys. Rev. D 92, 124010 (2015)

53. V. Iyer, R.M. Wald, Phys. Rev. D 50, 846 (1994)

54. K. Hotta, Y. Hyakutake, T. Kubota, H. Tanida, JHEP 0807, 066 (2008)

55. J.D. Brown, M. Henneaux, Commun. Math. Phys. 104, 207 (1986)

56. M.I. Park, J. Ho, Phys. Rev. Lett. 83, 5595 (1999)

57. M.I. Park, Nucl. Phys. B 634, 339 (2002)

58. S. Carlip, J. Korean Phys. Soc. 28, S447 (1995)

59. S. Carlip, Phys. Rev. Lett. 82, 2828 (1999)

60. J.A. Cardy, Nucl. Phys. B 270, 186 (1986)

61. H.W.J. Blote, J.A. Cardy, M.P. Nightingale, Phys. Rev. Lett. 56, $742(1986)$ 